\title{
Aerosol size distributions, retrieved from multi-band transmissometer data in the Southern Baltic Sea during the VAMPIRA trials
}

\author{
Arie N. de Jong, Alexander M.J. van Eijk, Marcel M. Moerman, Leo H. Cohen \\ TNO-Defense and Security, The Hague, The Netherlands
}

\begin{abstract}
In an earlier paper [1], data from our Multi-Band Radiometer Transmissometer (MSRT) were used to compare the ratio of extinction coefficients in different spectral bands during periods of changing visibility conditions. This ratio is an indication of the characteristics and origin (eg rural or maritime) of the haze- or fog particles, present in the measurement path. In this paper we will analyze the VAMPIRA transmission data in more detail by separating the contributions due to molecular extinction, scattering and (potentially) refraction. In our analysis we take the contribution due to scattering in order to obtain the characteristics of the Particle Size Distribution (PSD). For this purpose we take the average value and the slope of the measured transmission level in two neighboring spectral bands. Via a special simulation tool, developed for Junge-type PSD's, the slope of the PSD (defined: Junge exponent) and its value at a particle diameter of $1 \mu \mathrm{m}$ (Junge coefficient) can be determined via a set of retrieval steps. Reference is made to a similar approach [2] where in stead of a Junge distribution, three contiguous lognormal distributions are taken. The associated procedure for the Junge-type PSD is explained in detail in this paper and applied to the VAMPIRA transmission data. The versatility of the new retrieval method is demonstrated, especially when wavelengths around $1 \mu \mathrm{m}$ are chosen (a somewhat higher number than the diameter of the majority of the particles, so that most of the scattering is in the so-called Rayleigh regime). It is obvious, that the method fails in conditions of dense fog, when the transmission levels (average value and slope) over the $8.6 \mathrm{~km}$ path approach zero. The results are compared with in-situ PSD measurements, carried out simultaneously with a PMS (Particle Measurement System) probe at the pier near the Sürendorf shore station. In many conditions different results appear due to the fact that the MSRT system delivers path integrated data, while the PMS probe measures locally in a small volume. The MSRT data, collected over an overseas path, are more relevant to be used in the data analysis of the shore based sensor systems [3], measuring simultaneously signal values of distant point targets. The MSRT system has a higher signal to noise ratio and due to the shorter time constant, rapid fluctuations in particle characteristics are observed, not measured by the PMS probe. The availability of reliable aerosol characteristics (i.e. Junge exponent and -coefficient) allows a more precise interpretation of the data from the surveillance systems.
\end{abstract}

KEYWORDS: atmospheric transmission, aerosols, scattering, particle size distribution

\section{INTRODUCTION}

The atmospheric extinction of optical and infrared radiation by aerosols is determined by their scattering and absorption characteristics. In relation to this, the Particle Size Distribution (PSD) and the chemical composition (complex refractive index) of the particles are the basic parameters, which need to be known to predict the extinction coefficient and thus the atmospheric transmission for a given path-length. Figure 1 shows two PSD's for different visibility conditions, measured by the PMS probes, installed by TNO at the pier in Sürendorf during the VAMPIRA trials. The plot on the left is typical for good visibility conditions, while the plot on the right concerns a moderate to poor visibility condition. Both $\log -\log$ plots of $\mathrm{dN} / \mathrm{dD}$ versus $\mathrm{D}$ show a linear regression line, of which the slope in both cases is about the same, but the vertical position in the right plot is about a factor 10 higher. On the horizontal axis the diameter $\mathrm{D}$ of the particles is running from 0.2 to $20 \mu \mathrm{m}$. The plots show, that the number of particles for a diameter of $3 \mu \mathrm{m}$ is of the order of $10^{-1}$ to $10^{-2}$ per $\mu \mathrm{m}$ per $\mathrm{cm}^{3}$, which is really a low value, because this means, that integrated over the path-length of $8.6 \mathrm{~km}$, the totally projected area of all particles per $\mathrm{cm}^{2}$ becomes $0.6^{*} 10^{-2}$ to $0.6 * 10^{-3} \mathrm{~cm}^{2}$. This implies that the contribution for this small number of particles can be neglected for our measurement range. For small particles ( $D=0.2 \mu \mathrm{m}$ and smaller), the size becomes smaller than the wavelengths of the spectral bands of optical and IR sensors (and our MSRT system). For these particles the dominant scattering mechanism obeys Rayleigh's $1 / \lambda^{4}$ law and can generally be neglected. 

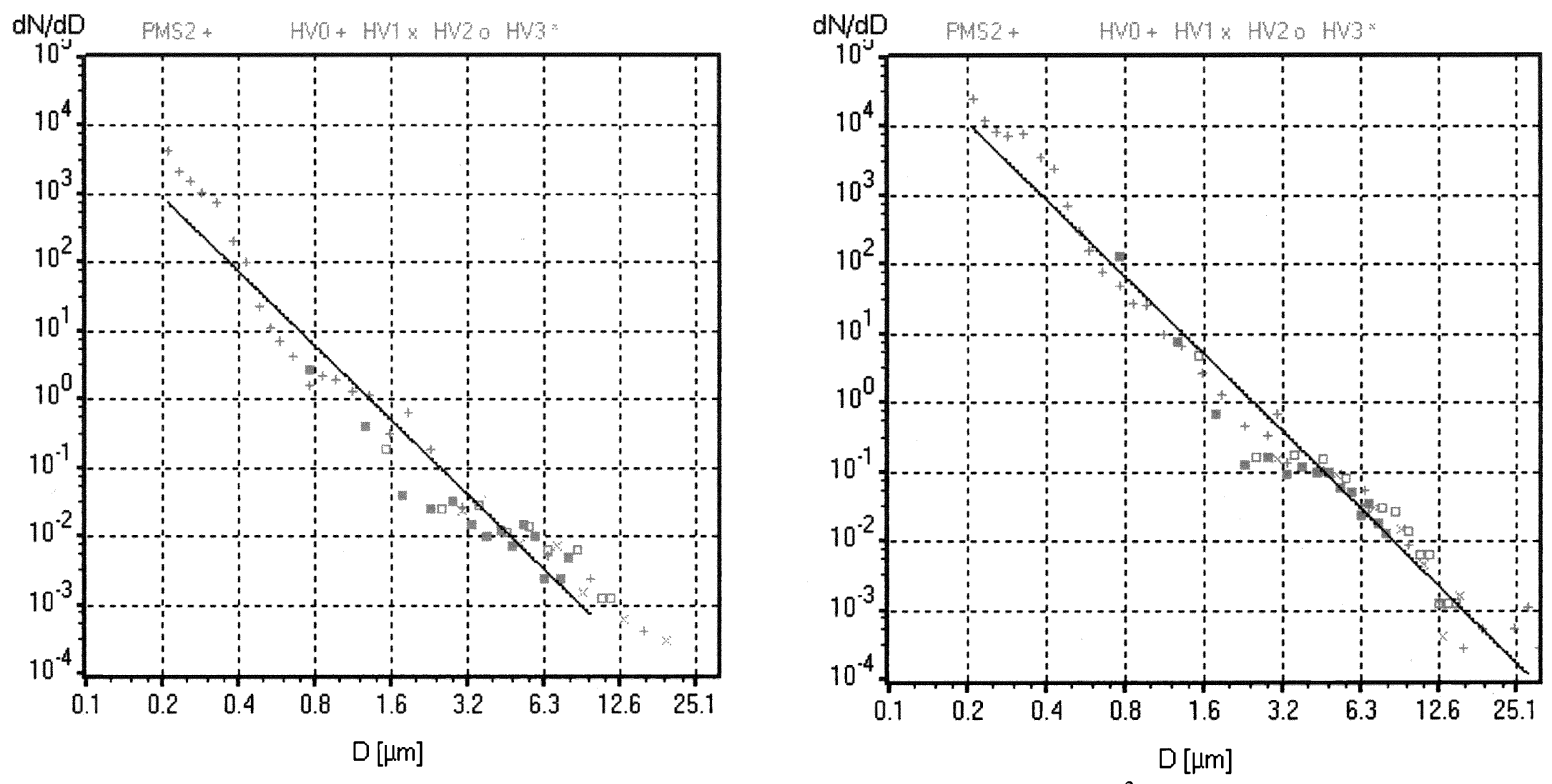

Figure 1. Example of different PSD's (dN/dD) in number of particles per $\mu \mathrm{m}$ per $\mathrm{cm}^{3}$ during the VAMPIRA trials. Left: 26 March 2004, 17.36 (UTC), good visibility; Right: 31 March 2004, 23.01 (UTC), poor visibility.

For particles with intermediate sizes, we have to consider the relation between the extinction efficiency factor $\mathrm{Q}_{\mathrm{ext}}$ and $\mathrm{x}=\pi \mathrm{D} / \lambda: \mathrm{Q}_{\mathrm{ext}}=2-4 * \sin (0.66 \mathrm{x}) /(0.66 \mathrm{x})+4 *\{1-\cos (0.66 \mathrm{x})\} /\left\{(0.66 \mathrm{x})^{2}\right\}$, where $\lambda$ denotes the wavelength, as shown in Figure 2 (left, see eg [4]). The plot is valid for a non absorbing water droplet with a refractive index of 1.33 . It is noted, that $\mathrm{Q}_{\mathrm{ext}}$ is directly coupled to the extinction cross section $\sigma_{\mathrm{ext}}$ by the relation: $\sigma_{\mathrm{ext}}=\mathrm{Q}_{\mathrm{ext}}{ }^{*} \pi \mathrm{D}^{2} / 4$. The figure shows, that

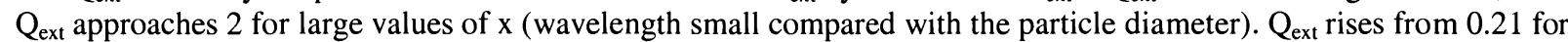
$\mathrm{x}=1$ to 2.9 for $\mathrm{x}=5$, which implies for a wavelength of $1 \mu \mathrm{m}$ an increase in particle diameter from about 0.3 to $1.5 \mu \mathrm{m}$. For bigger particles $Q_{\text {ext }}$ reaches a maximum value for $x=6$ and starts to decrease to a minimum value of about 1.6 for $\mathrm{x}=12$ (D about $4 \mu \mathrm{m})$. As we noted before the total number of particles in this size range is low, so most of the scattering in our case is in the range below the first maximum, where $\mathrm{Q}_{\mathrm{ext}}$ can be approximated by $\mathrm{Q}_{\mathrm{ext}}=0.68^{*} \mathrm{x}-0.47$. Taking the original formula for $\mathrm{Q}_{\mathrm{ext}}$, we can perform a direct calculation of the total extinction cross section or extinction coefficient in $\mathrm{km}^{-1}: \sigma(\lambda)=\int \mathrm{Q}_{\mathrm{ext}}{ }^{*}(\mathrm{dN} / \mathrm{dD})^{*}\left(\pi \mathrm{D}^{2} / 4\right) * \mathrm{dD}$ for all particles in the distribution with a diameter between $\mathrm{D}_{1}$ and $\mathrm{D}_{2}$. An example of such calculations is shown in Figure 2 (right), where $\sigma(\lambda)$ is plotted as a function of the slope of the PSD for five wavelengths in the visible and near IR, indicating the viability of a retrieval method based on transmission data.
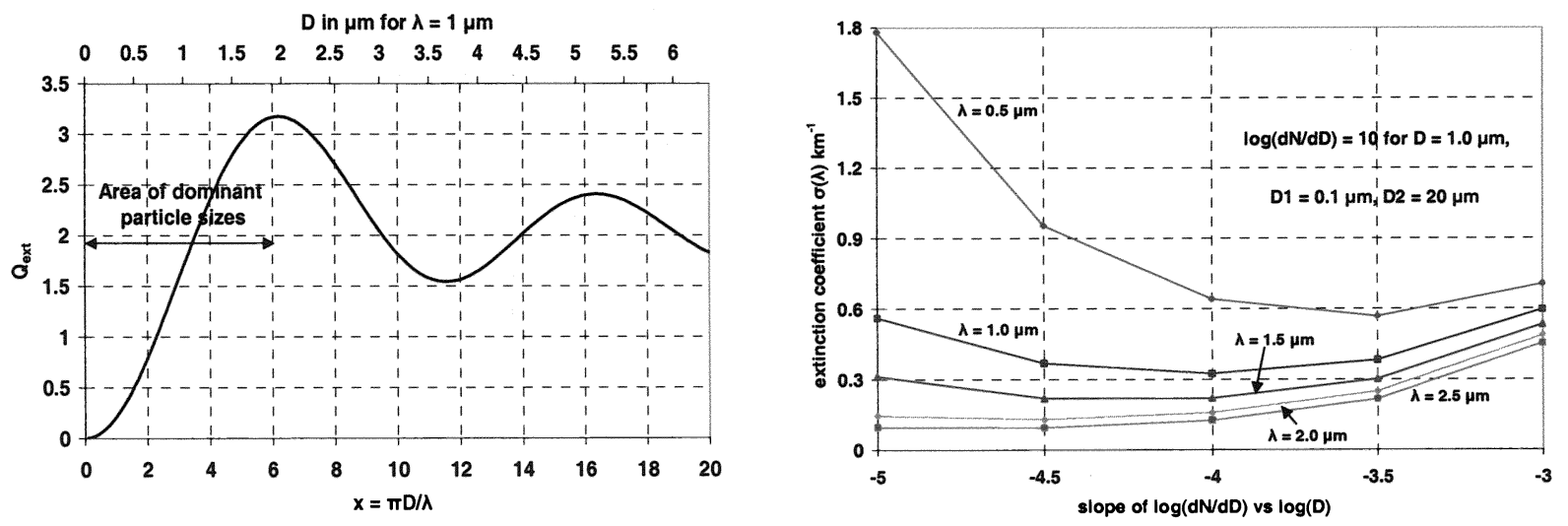

Figure 2. Relation between the efficiency factor $\mathrm{Q}_{\mathrm{ext}}$ and the ratio $\pi \mathrm{D} / \lambda$ for a particle with a refractive index of 1.33 (left) and plots of $\sigma(\lambda)$ versus the slope of the PSD for five wavelengths ranging from 0.5 to $2.5 \mu \mathrm{m}$. 
In the previous paper on the VAMPIRA transmission data we compared the ratio of the extinction coefficient in one spectral band and that in another band during conditions of changing visibility. It was somewhat surprising, that the plots were straight lines, indicating that the ratio was about constant in these conditions. It was concluded, that in these cases the slope of the PSD remained the same, but that just the particle density (concentration) varied. One aspect of that type of analysis was that the absolute value of the transmission was not relevant, as long as the mutual responsivities in the different channels was correct. As a result, by comparing the various ratio's with those in MODTRAN, we could draw conclusions about the nature of the aerosols: urban or maritime. In this paper, the transmission data are recalibrated and absolute transmission data become available. Next we separate the transmission values, just caused by scattering. These data are taken in order to retrieve the PSD's during the VAMPIRA trials period. Finally the retrieved data are compared with those from the shore based PMS instrument. In the next chapter we will start with an explanation of the calculation tool for Junge-type PSD's, leading towards the retrieval methodology.

\section{THE CALCULATION TOOL}

A simple tool has been developed, allowing the calculation of scattering and extinction coefficient for a Junge-type PSD: $\log (\mathrm{dN} / \mathrm{dD})=\mathrm{J}_{\mathrm{c}}+\mathrm{J}_{\mathrm{e}}{ }^{*} \log (\mathrm{D})$ or $\mathrm{dN} / \mathrm{dD}=10^{\mathrm{Jc}} * \mathrm{D}^{\mathrm{Je}}$. The parameters $\mathrm{J}_{\mathrm{e}}$ and $\mathrm{J}_{\mathrm{c}}$ are called Junge exponent respectively $\mathrm{Junge}$ coefficient. Figure 3 shows some typical cases of this type of distribution for constant $J_{e}$ and constant $J_{c}$. The scattering and extinction coefficient (in $\mathrm{km}^{-1}$ ) are calculated on the basis of the extinction cross section $\sigma(\lambda)$ mentioned before by counting all particles in a path length of $1 \mathrm{~km}$. In our calculations we limit ourselves to the wavelengths of the MSRT, listed in Table 1, together with some other parameters. The calculation tool provides the extinction/scattering coefficient for the center wavelength of each spectral band. This result is of course an approximation of the extinction/scattering coefficient for the whole band (in $\mathrm{km}^{-1}$ ), as scattering in some cases is strongly wavelength dependent.

Table 1. List of technical parameters of the MSRT transmissometer during VAMPIRA and recalibration data

\begin{tabular}{|c|c|c|c|c|c|c|c|}
\hline Channel & Spectral band & Center & \multicolumn{2}{|c|}{ Receiver optics } & $\tau_{\text {mol }}$ & $\tau_{\text {mol }} * \tau_{\text {aer }}$ & $\tau_{\text {aer }}$ \\
\hline No & $\mu \mathrm{m}$ & $\mu \mathrm{m}$ & FOV(mr) & Pupil $(\mathrm{mm})$ & No aer. & $80 \mathrm{~km}$ vis. & No mol. \\
\hline 1 & $0.40-0.49$ & 0.45 & 73 & $20 \times 20$ & 0.769 & 0.520 & 0.676 \\
\hline 2 & $0.57-0.65$ & 0.61 & 77 & $10 \times 10$ & 0.922 & 0.692 & 0.751 \\
\hline 3 & $0.78-1.04$ & 0.91 & 77 & $10 \times 10$ & 0.839 & 0.707 & 0.843 \\
\hline 4 & $1.39-1.67$ & 1.53 & 86 & $10 \times 10$ & 0.599 & 0.555 & 0.927 \\
\hline 5 & $2.12-2.52$ & 2.32 & 83 & $10 \times 10$ & 0.749 & 0.716 & 0.956 \\
\hline 6 & $3.55-4.15$ & 3.85 & 16 & $55 \Phi$ & 0.775 & 0.749 & 0.967 \\
\hline 7 & $7.8-13.7$ & 10.7 & 27 & $75 \Phi$ & 0.641 & 0.624 & 0.974 \\
\hline
\end{tabular}
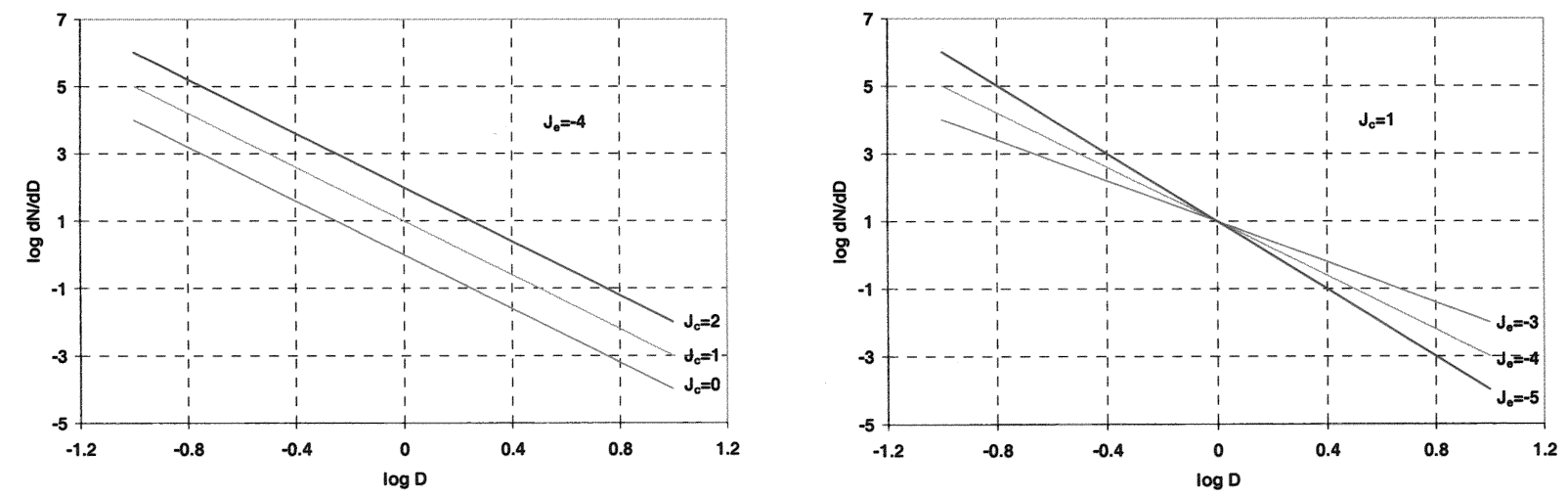

Figure 3. Two cases of Junge-type PSD: constant exponent $\mathrm{J}_{\mathrm{e}}$ (left) and constant coefficient $\mathrm{J}_{\mathrm{c}}$ (right) 
A set of calculations has been carried out of the extinction coefficient for all center wavelengths of the MSRT, including for the average wavelengths between channels 2 and 3: $0.76 \mu \mathrm{m}$ and channels 3 and 4: $1.22 \mu \mathrm{m}$. For the visual and near IR channels (1-5), the extinction coefficient and the scattering coefficient are nearly the same, as the refractive index of water has no complex component in these bands. The result for five of the wavelengths is shown in Figure 4 (left) for five values of the Junge exponent. It is interesting to consider the behavior of the transmission level, caused by aerosols, along an $8.6 \mathrm{~km}$ path (the path-length at Sürendorf). For this purpose calculations were carried out with various levels of $\mathrm{J}_{\mathrm{c}}$ in such a way, that the average transmission (transmission at the average wavelengths 0.76 and $1.22 \mu \mathrm{m}$ ) was constant but variable from 0.03 to 0.90 . The result is shown in Figure 4 (right) for three values of $J_{e}$ and for the wavelength of $1.22 \mu \mathrm{m}$. We obtain from the plots an impression on the degree of linearity of the slopes and the slopes themselves for various transmission levels. It is noted, that in all calculations we ignore particles with $\mathrm{D}<0.1$ and $\mathrm{D}>20 \mu \mathrm{m}$.
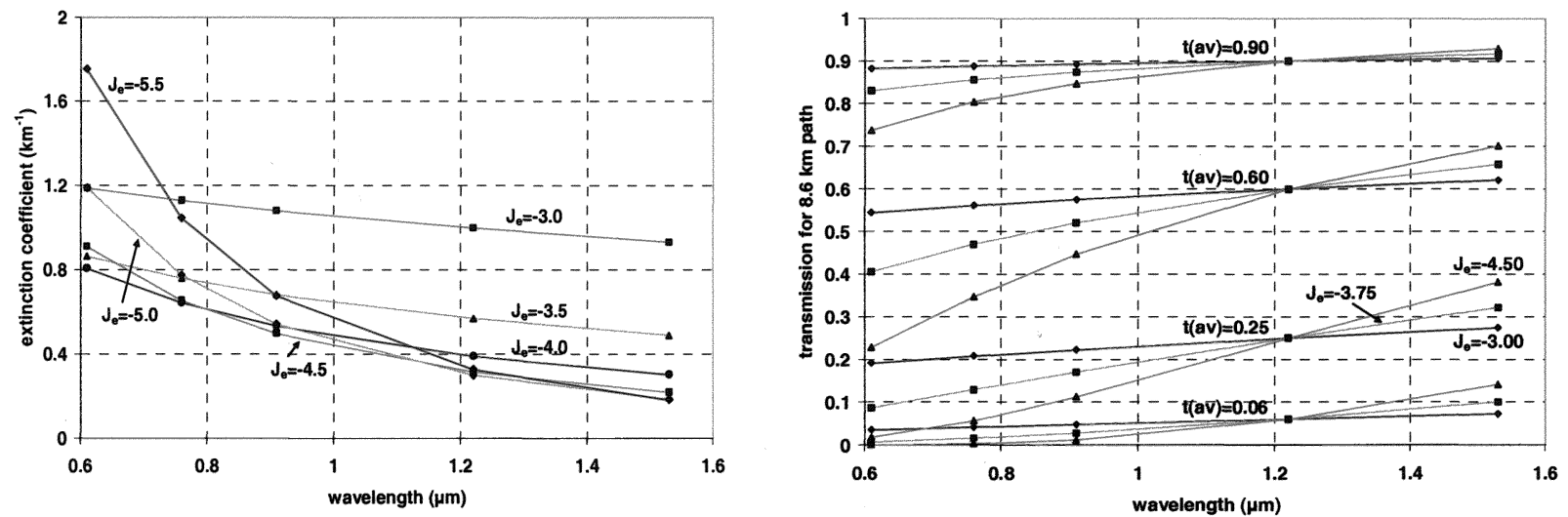

Figure 4. Plots of extinction coefficient versus wavelength for $\mathrm{J}_{\mathrm{c}}=2.50$ and various values of $\mathrm{J}_{\mathrm{e}}$ (left) and of transmission along an $8.6 \mathrm{~km}$ path versus wavelength at various average transmission levels $\mathrm{t}(\mathrm{av})$ and $\mathrm{J}_{\mathrm{e}}$ (right).

Another interesting issue is the ratio of the extinction coefficients at various wavelengths, as these ratio's are independent of the value of $J_{c}$. The result of three ratios as a function of $J_{e}$ is shown in Figure 5 (left). We find that for values of Je below 3.0, all ratios approach the value of 1 , which is what we might expect as the Junge distribution obtains a smaller slope, implying a higher number of bigger particles compared to the number of smaller particles. The scattering enters the right part of the $Q_{\text {ext }}$ plot in Figure 2. The effect of variable $J_{c}$ at constant level of $J_{e}$ is shown for two wavelengths in Figure 5 (right). The plots show, that the behavior follows an S-shaped curve, nearly independent of the value of Je and of the wavelength. We find that the plots for the three $\mathrm{J}_{\mathrm{e}}$ levels for $0.61 \mu \mathrm{m}$ are located more closely together than those for $1.53 \mu \mathrm{m}$. We conclude that small changes in $\mathrm{J}_{\mathrm{c}}$ level provide more variation in transmission at $0.61 \mu \mathrm{m}$. When the level of $\mathrm{J}_{\mathrm{c}}$ varies, the difference in transmission at the two wavelengths starts to increase, reaches a maximum, followed by a decrease, all occurring over a variation in $\mathrm{J}_{\mathrm{c}}$ of about 2 (a factor of 100 in number of particles).
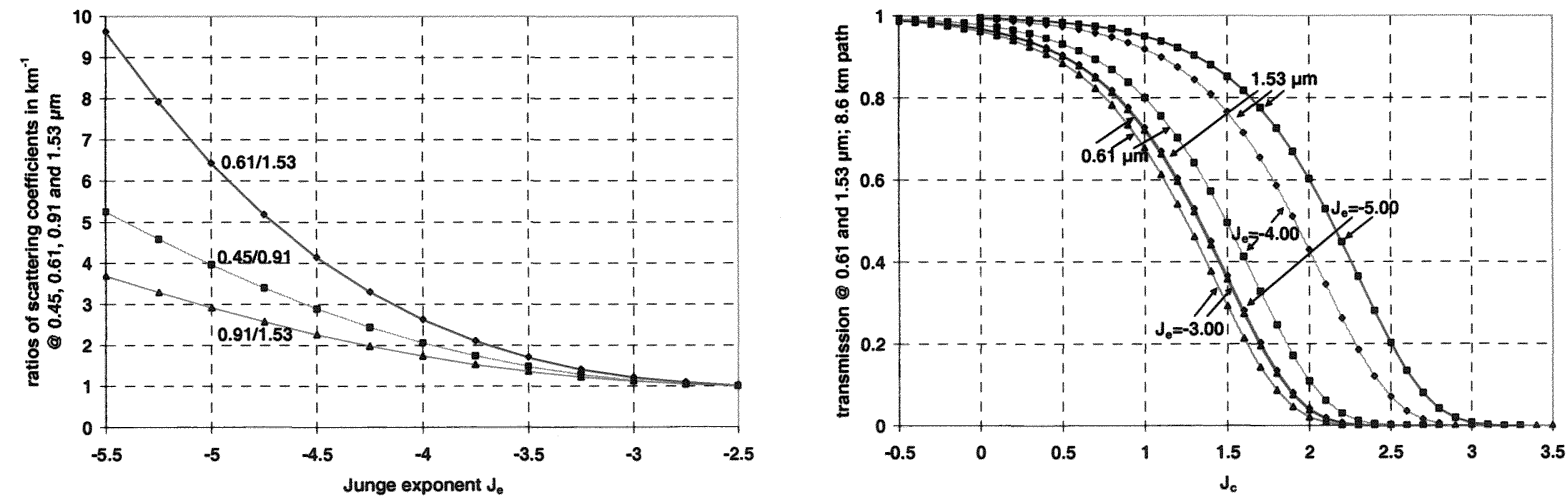

Figure 5. Plots of the ratio of extinction coeffients versus $J_{e}$ for various wavelengths (left) and of the transmission versus $\mathrm{J}_{\mathrm{c}}$ for two wavelengths $(0.61$ and $1.53 \mu \mathrm{m})$ and three values of $\mathrm{J}_{\mathrm{e}}$. 
Of great importance for the retrieval method is the relation between the slope $s(t)$ of the plot of the transmission versus wavelength and $\mathrm{J}_{\mathrm{e}}$. In the previous paper [2], we took three and four wavelengths due to the somewhat irregular set of log-normal PSD's. In this paper we take just two wavelengths: the combination of 0.61 and $0.91 \mu \mathrm{m}$ and the combination of 0.91 and $1.53 \mu \mathrm{m}$. The result for the latter set is shown in Figure 6 (left) for a number of average transmission levels $\mathrm{t}(\mathrm{av})($ at $1.22 \mu \mathrm{m})$ between 0.03 and 0.90 . The slopes $\mathrm{s}\left(\mathrm{J}_{\mathrm{e}}-\mathrm{t}\right)$ in these plots are approximated by a linear regression line, which are plotted versus $t(\mathrm{av})$ in Figure 6 (right). This inverse U-shaped curve is approximated by the formula:

$$
\mathrm{s}\left(\mathrm{J}_{\mathrm{e}}-\mathrm{t}\right)=-3.12 *\{\operatorname{tg}(90 * \mathrm{t}(\mathrm{av}))\}^{-0.5}-2.55^{*}\{\operatorname{tg}(90 * \mathrm{t}(\mathrm{av}))\}^{0.9}+1.6
$$

The value of Je is then retrieved by means of the relationship: $J_{e}=s\left(J_{e}-t\right) *_{s}(t)-2.64$, where $s(t)$ is the measured slope or: $s(t)=\{t(1.53)-t(0.91)\} / 0.62$. In order to retrieve $J_{c}$ we consider the plots of $J_{c}$ versus $t(a v)$ for a number of $J_{e}$ values. These plots, shown in Figure 7 (left) are approximated by the linear relationship: $J_{c}=-1.544 * t(a v)+d$, where the parameter $d$ is dependent on the value of $\mathrm{J}_{\mathrm{e}}$ as shown in Figure 7 (right). For $\mathrm{d}$ the following approximation has been found: $\mathrm{d}=-0.1476 * \mathrm{~J}_{\mathrm{e}}{ }^{2}-1.4479 * \mathrm{~J}_{\mathrm{e}}-0.8978$.

For the wavelengths 0.61 and $0.91 \mu \mathrm{m}$ with average wavelength $0.76 \mu \mathrm{m}$ similar calculations have been carried out, leading to the following retrieval formulae:

$$
\left.\mathrm{s}\left(\mathrm{J}_{\mathrm{e}}-\mathrm{t}\right)=-0.91 *\{\operatorname{tg}(90 * \mathrm{t}(\mathrm{av}))\}^{-0.71}-1.12 *\{\operatorname{tg}(90 * \operatorname{tav}))\right\}^{1.07}-0.61 \quad \text { with } \quad \mathrm{J}_{\mathrm{e}}=\mathrm{s}\left(\mathrm{J}_{\mathrm{e}}-\mathrm{t}\right) * \mathrm{~s}(\mathrm{t})-2.67
$$

and : $\mathrm{J}_{\mathrm{c}}=-1.544 * \mathrm{t}(\mathrm{av})+\mathrm{d}$, with $\mathrm{d}=-0.1653 * \mathrm{~J}_{\mathrm{e}}{ }^{2}-1.4157 * \mathrm{~J}_{\mathrm{e}}-0.7039$, which are definitely different from the formulae for the longer wavelength band around $1.22 \mu \mathrm{m}$, due to the different extinction coefficients in both areas.
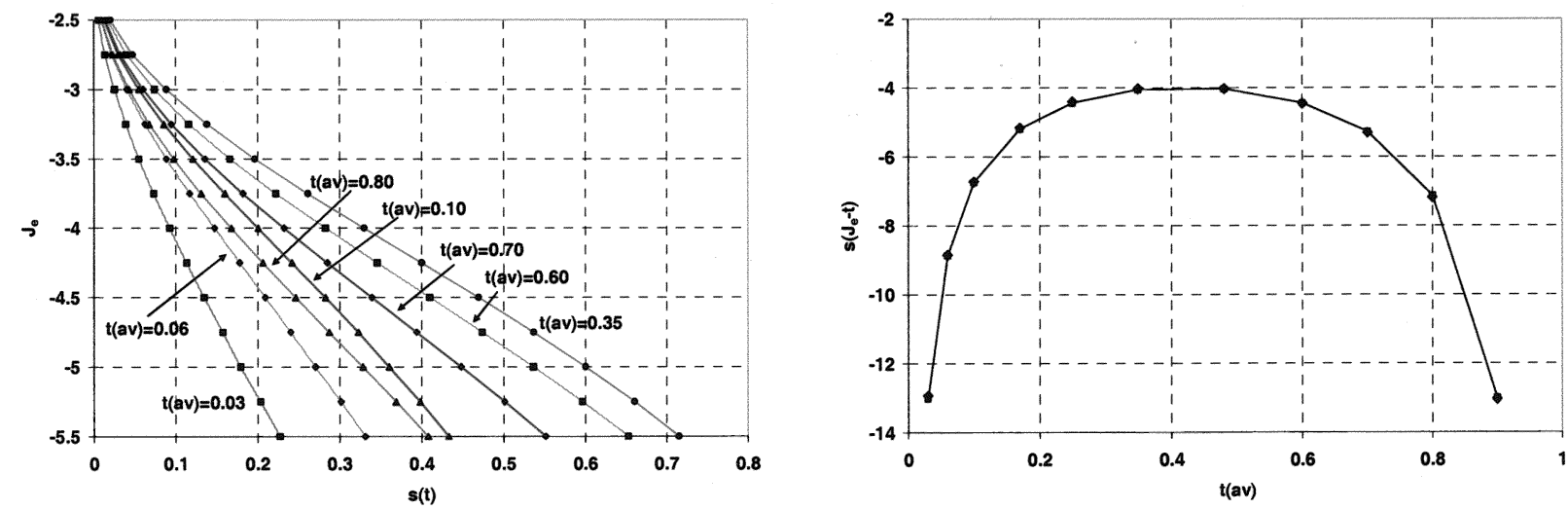

Figure 6. Plot of $J_{e}$ versus $s(t)$ for a set of $t(a v)$ values (left) and slope $s\left(J_{e}-t\right)$ versus $t(a v)$ for these plots (right)
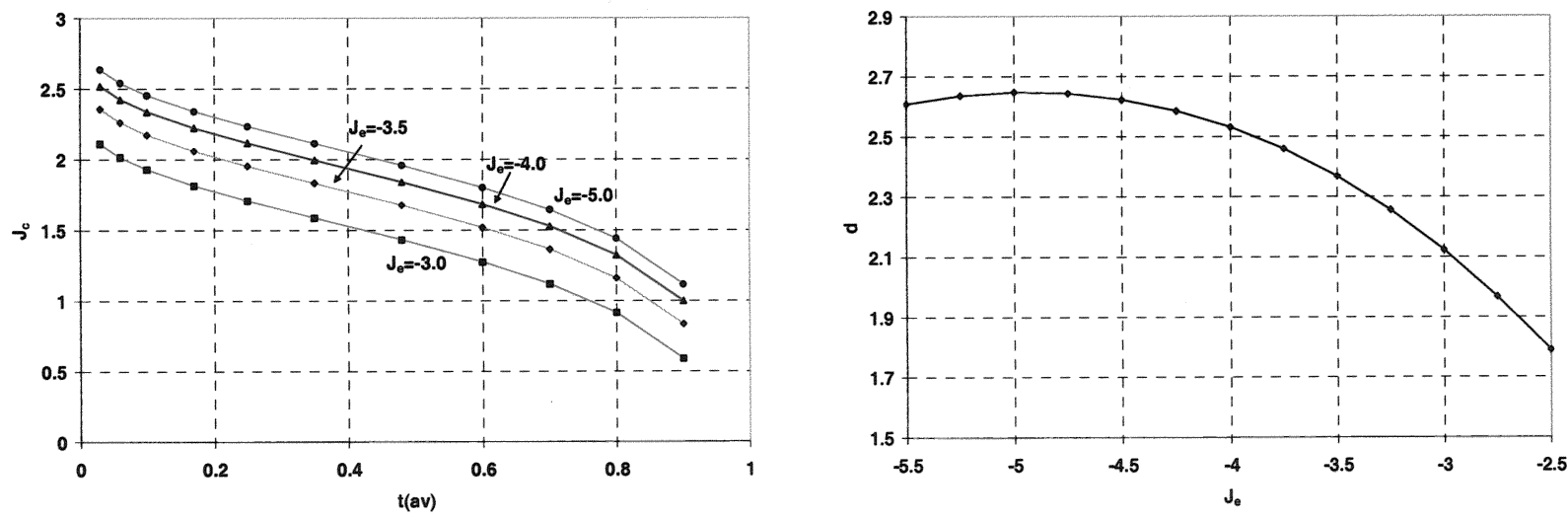

Figure 7. Plots of $J_{c}$ versus $t(a v)$ for a set of $J_{e}$ values (left) and relation between parameter $d$ and $J_{e}$ (right) 


\section{DATA RECALIBRATION}

In general a transmissometer is calibrated by collecting in the laboratory the radiant intensity properties of the transmitter and the responsivity characteristics of the receiver. In addition the system is tested over a limited range in order to allow full pupil filling for both ends [5]. During the preparation of VAMPIRA, the amount of available time was limited, so we performed an "after trial" method, based upon the signal levels during a short period of extremely good visibility and low absolute humidity. From the uncalibrated transmission data and the data from the Nephelometer and the PMS system (as shown in Figure 8), it was found that the maximum signal occurred on 26 March around 18.00 (UTC). We did run the MODTRAN model [6] for these conditions and used the outcome as basis for the "calibrated" transmission data.
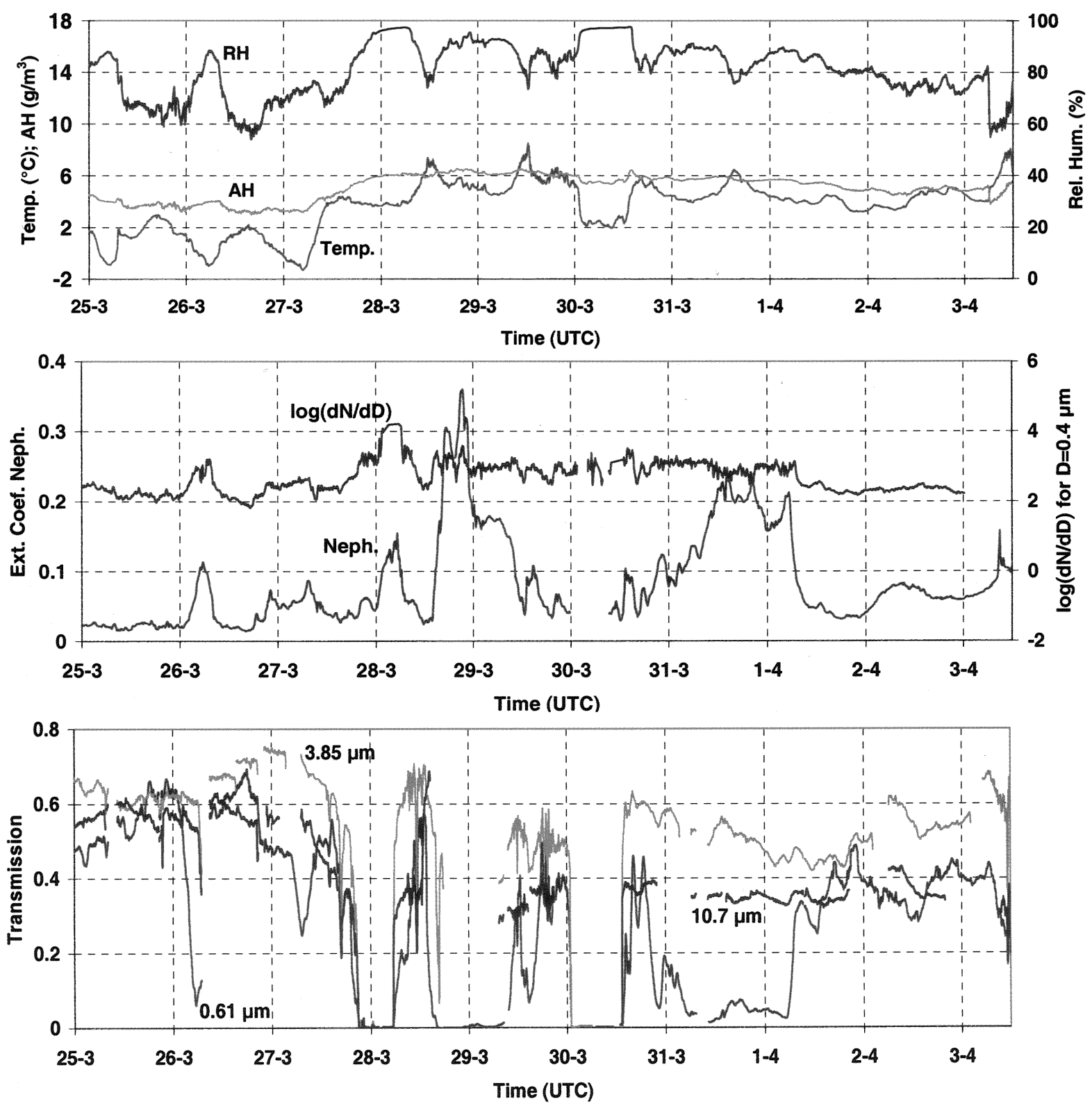

Figure 8. Weather data from the mid-path buoy (top), ext. coeff. from Nephelometer and $\log (\mathrm{dNdD})$ for $\mathrm{D}=0.4 \mu \mathrm{m}$ from PMS system (middle) and recalibrated transmission data for $0.61,3.85$ and $10.7 \mu \mathrm{m}$ from MSRT (bottom) 
The estimated transmission levels for molecular extinction, aerosol extinction and both extinction combined are listed in Table 1 for each of the channels for the $8.6 \mathrm{~km}$ path. An estimation was made for the potential contribution of refractive gain, as the Air to Sea Temperature Difference (ASTD) was sometimes negative during the first days of the trial. It was found however, that for the relatively short path and the high level above the water (receiver and transmitter at 8 meter or more above the water), including the temperature profile collected with the buoy sensors, the contribution from refractive gain was less than $10 \%$ at the selected time of maximum signal. The result for three of the seven channels is shown in the bottom plots of Figure 8, where gaps occur in the 4 and $10 \mu \mathrm{m}$ channels due to the non-cooling status of their Liquid Nitrogen dewars. The data show that some periods with dense fog with poor visibility did occur on 28, 29 and 30 March. In the plots from the Nephelometer and the PMS system, the extinction coefficient $\left(\mathrm{km}^{-1}\right)$ respectively the number particles as $\log (\mathrm{dN} / \mathrm{dD})$ for a particle diameter of $0.4 \mu \mathrm{m}$ are shown.

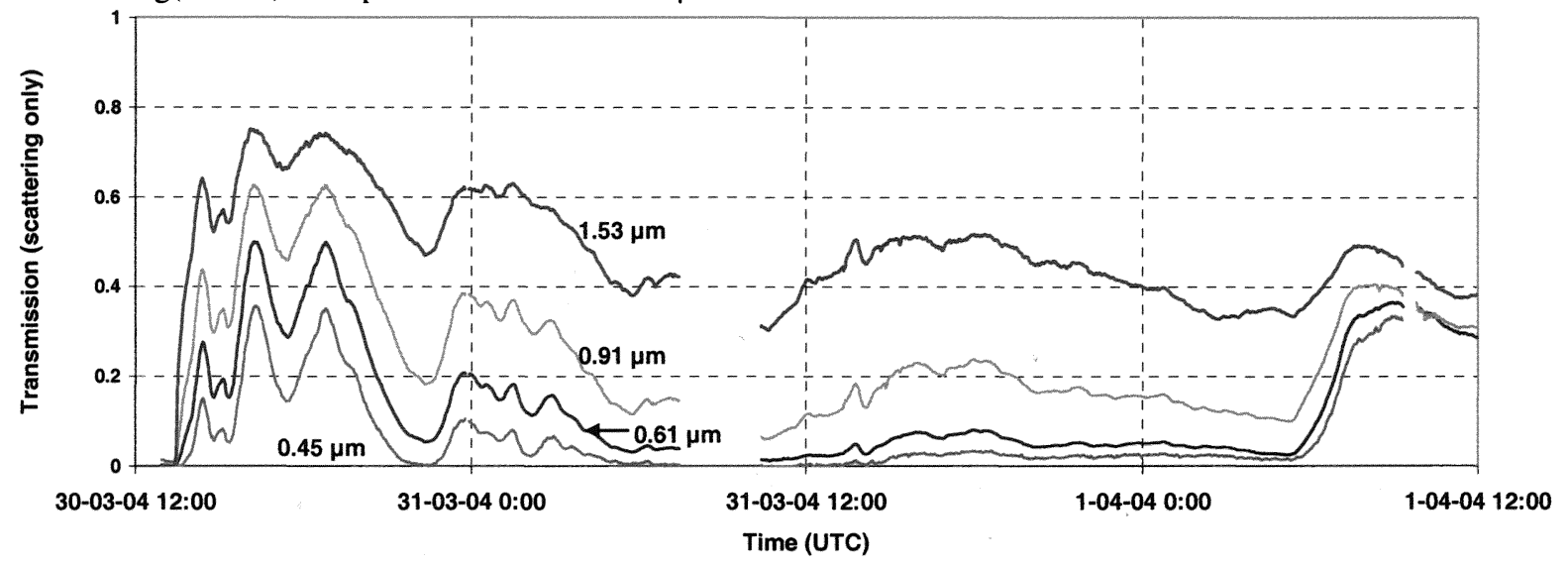

Figure 9. Transmission data (scattering only) for channels 1-4 for 30 March 12.00 to 1 April 12.00

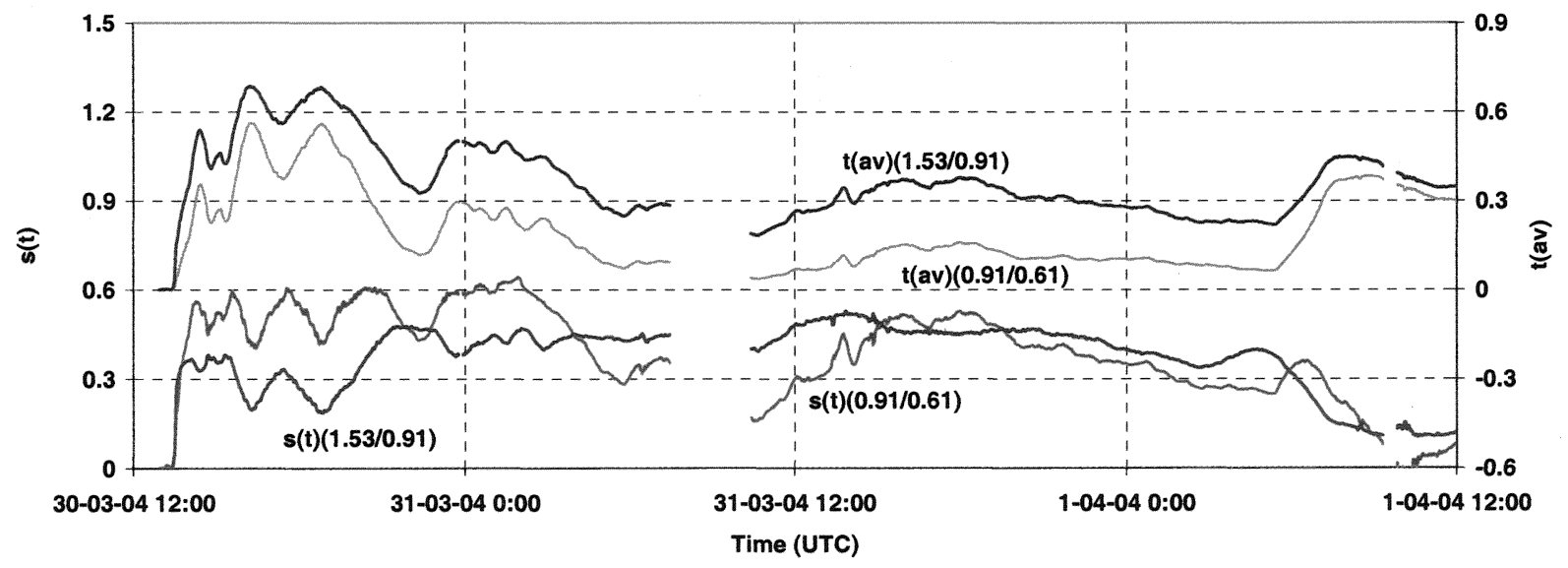

Figure 10. Behavior of $s(t)$ and $t(a v)$ for data of Figure 9 for channels $2+3$ and $3+4$

We did sub-divide the total measurement period in four smaller periods of about two days. Transmission data for the third period (scattering only), after removal of the molecular extinction, are plotted in Figure 9 for the channels 1-4 with shortest wavebands. The data are averaged over a period of five minutes in order to reduce short term signal fluctuations due to scintillation. The data show an interesting variety of transmission values for all four bands, enabling meaningful testing of the new retrieval method. For some short periods, the transmission shows rapid fluctuations, probably because of the passage of aerosol (hazea) clouds. Figure 10 shows for the data of Figure 9 the variations of the transmission slopes $s(t)$ and average transmissions $t(a v)$ with time. It is clear, that in case of small values of $t(a v)$, such as around 12.00 on the $31^{\text {st }}$ of March, $\mathrm{s}(\mathrm{t})$ is becoming small and $\mathrm{s}\left(\mathrm{J}_{\mathrm{e}}-\mathrm{t}\right)$ is getting a large negative value. This leads to greater errors in the retrieved values of $J_{e}$ and $J_{c}$ than in cases of moderate $t(a v)$ conditions. 

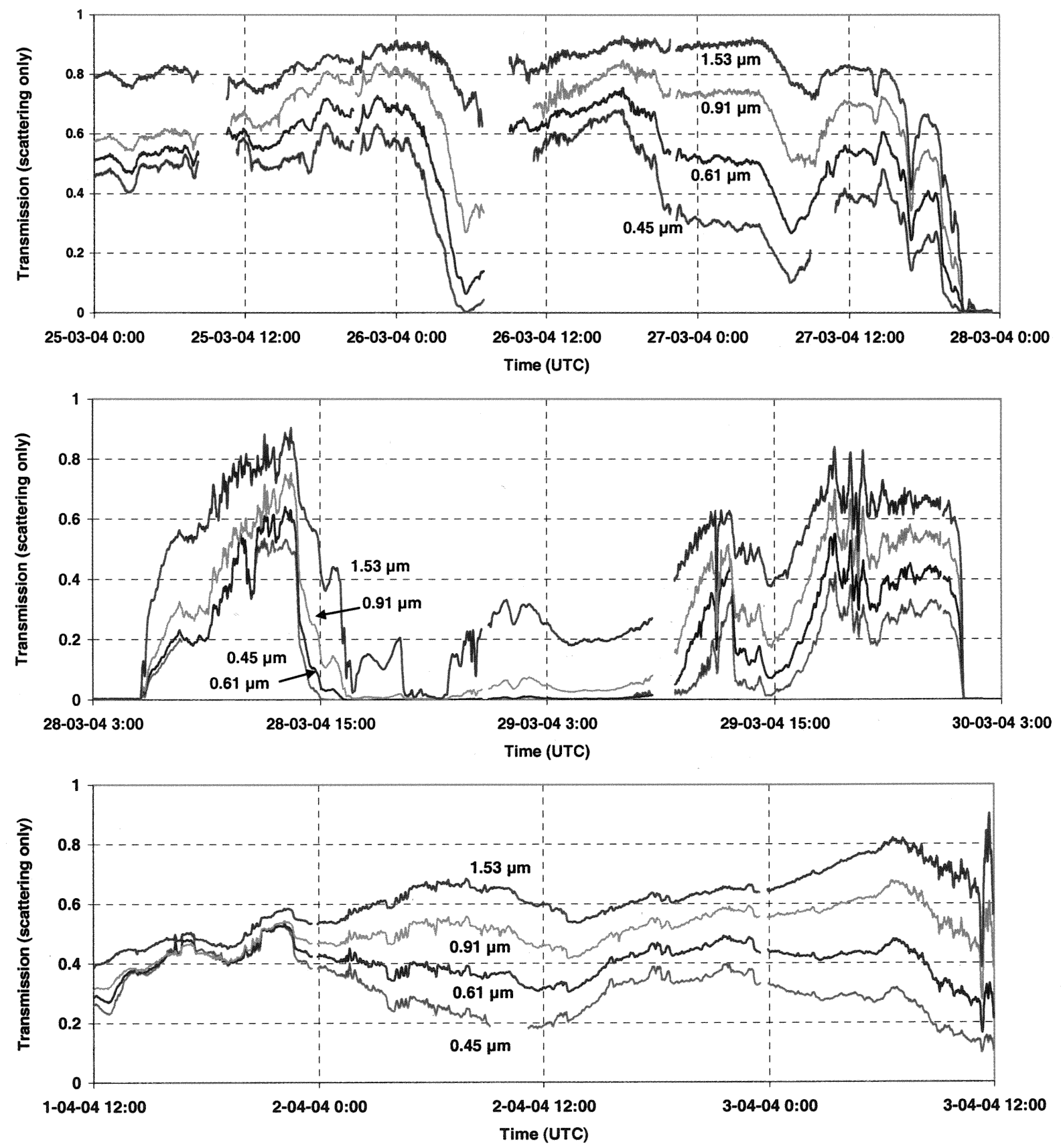

Figure 11. Remaining transmission data for channels 1-4 for 25 March to 3 April 2004

The remaining transmission data for the other sub-periods are shown in Figure 11. In some occasions we find rapid fluctuations in transmission values, even after five minutes averaging. One reason for these fluctuations may be short duration rain showers somewhere mid-path. Another reason is the presence of sea and sky-glint in the morning periods after 08.00 (UTC), entering the optics of the receiver and causing signal instabilities. It is interesting to note that in the period from 28 March-17.00 to 29 March-10.00, the transmission for channels 1 and 2 is practically zero, while the transmission for the $1.53 \mu \mathrm{m}$ band stays at a value of about 0.2 . 


\section{PSD RETRIEVAL}

Following the procedure, described in section 2, we calculated from the transmission data the slopes $s(t)$ and the average $\mathrm{t}(\mathrm{av})$ for the two sets of two wavelengths: $0.91+0.61 \mu \mathrm{m}(1)$ and $1.53+0.91 \mu \mathrm{m}(2)$. From these results the parameters of Junge distributions were retrieved: $\mathrm{J}_{\mathrm{e}}(1)$ and $\mathrm{J}_{\mathrm{c}}(1)$ respectively $\mathrm{J}_{\mathrm{e}}(2)$ and $\mathrm{J}_{\mathrm{c}}(2)$. For the three most interesting sub-periods the results are shown in Figure 12 together with the values of $J_{e}$ and $J_{c}$, determined with the PMS system. We did omit the results for the period with very poor visibility on the $28^{\text {th }} / 29^{\text {th }}$, because of the failure of the retrieval methodology for this kind of conditions. A comparison can be made between the retrieved data for bands (1) and bands (2) and data from
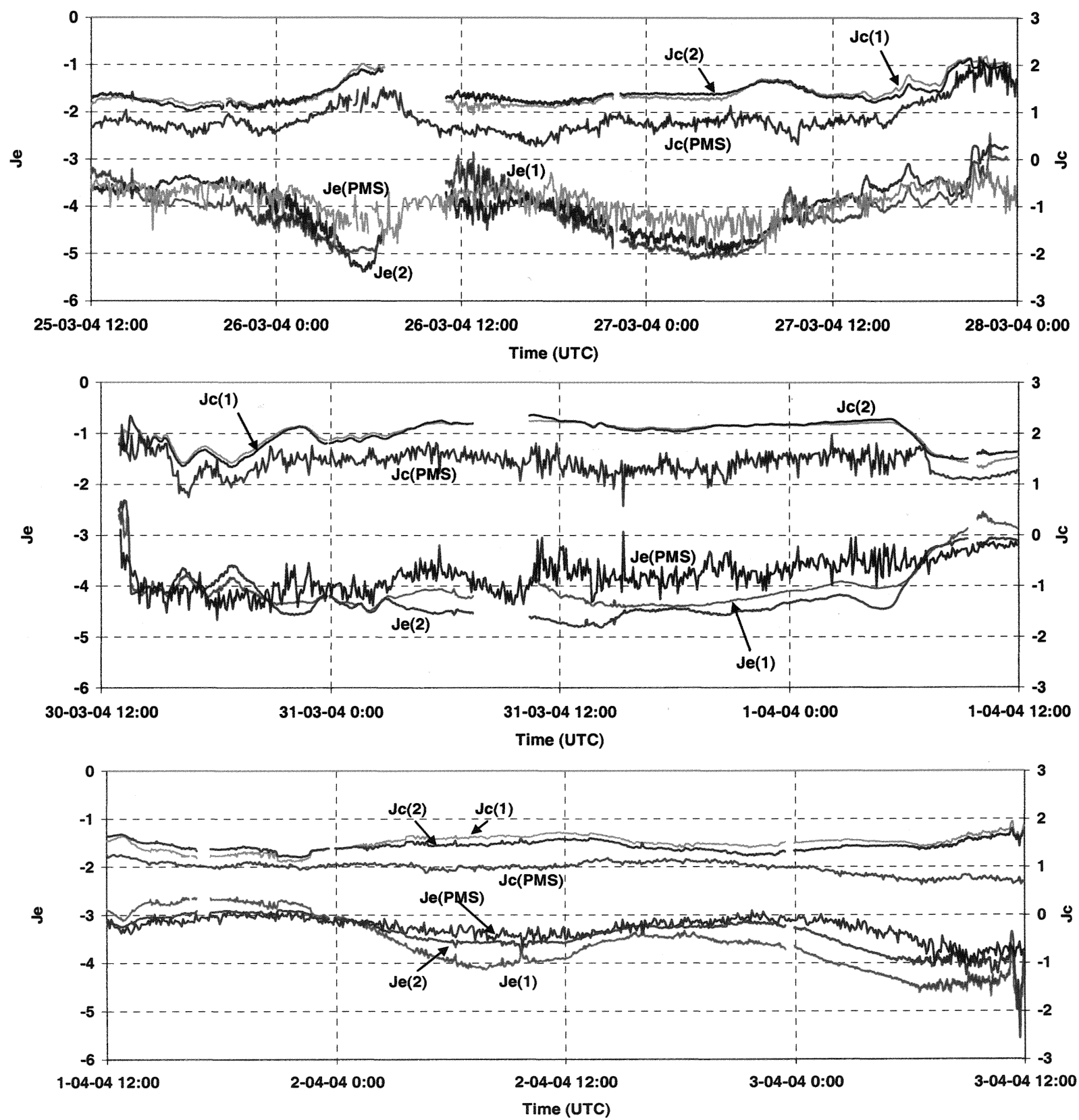

Figure 12. Values of Je and Jc, retrieved from MSRT data and measured by PMS system 
the PMS system. First of all we find a much better signal to noise ratio for the data, retrieved from the transmission data. As a result rapid fluctuations in the particle density (eventually somewhere else in the measurement path) can be followed more easily. Secondly the agreement is quite good, although the value of $\mathrm{J}_{\mathrm{e}}$, obtained from the PMS system, tends to be higher than those of the MSRT system. One reason is the way how the slope is determined from the particle data (see Figure 1), where the bigger particles are taken too much into account. Another comparison is that of the number of particles, as measured with the MSRT and PMS systems. The result is shown in Figure 13 for three of the sub-periods, where we compared $\log (\mathrm{dN} / \mathrm{dD})$ for a particle diameter of $0.4 \mu \mathrm{m}$ from bands (2) of the MSRT with the data from the polynome through the PSD measurements of the PMS system.
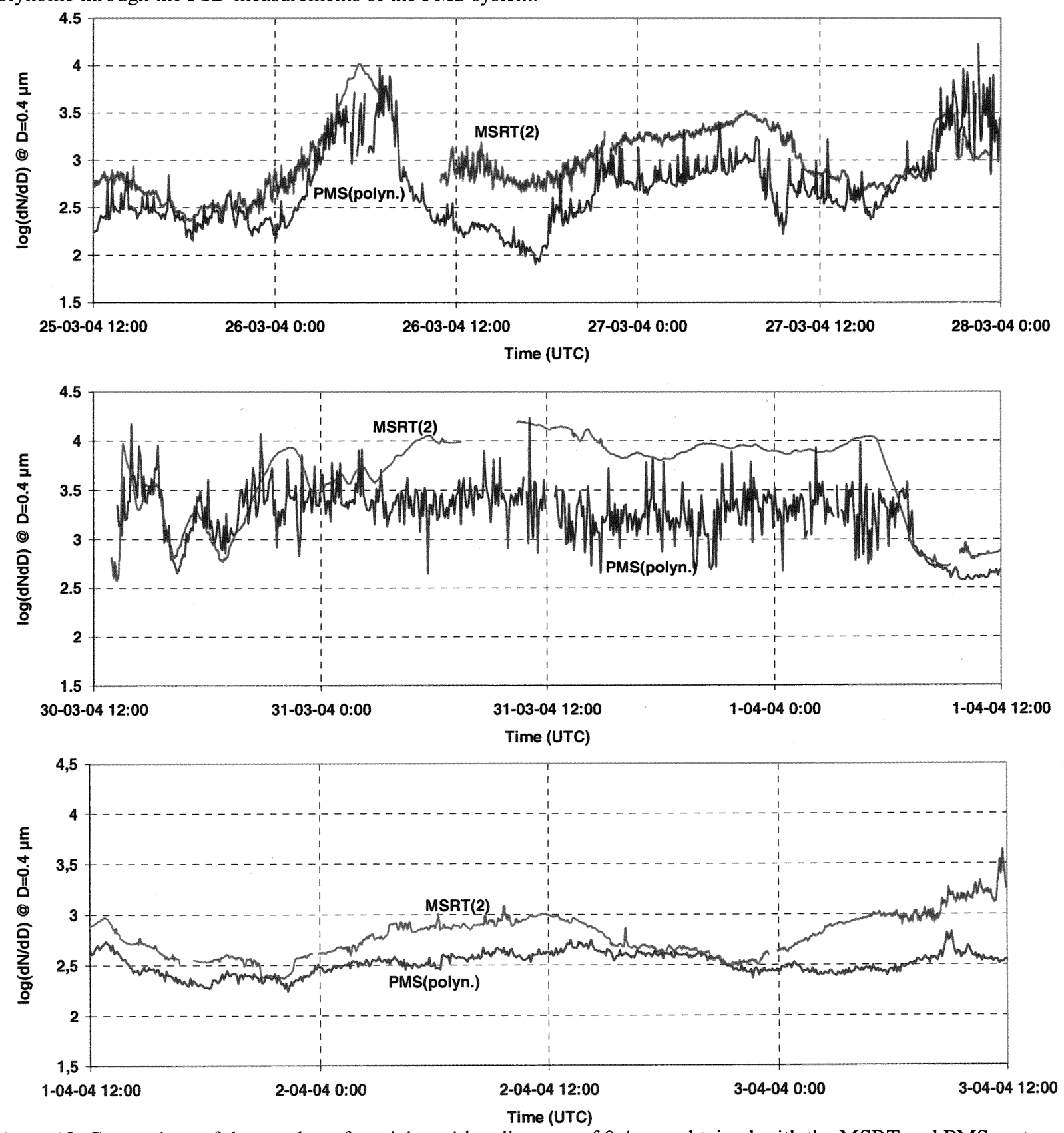

Figure 13. Comparison of the number of particles with a diameter of $0.4 \mu \mathrm{m}$, obtained with the MSRT and PMS systems 
The data show for some periods a very good agreement with differences in $\log (\mathrm{dN} / \mathrm{dD})$ of less than 0.1 (factor 1.25$)$. The maximum deviation is about 0.6 (factor 4). If we would obtain the particle density for the PMS system from its Je and Jc values, the deviation would have been greater, because most of the time the Junge regression line stays below the real number of particles for particle diameters of $0.4 \mu \mathrm{m}$ (see again Figure 1).

A final comparison has been made for the particle density $\log (\mathrm{dN} / \mathrm{dD})$, retrieved with bands (1) $(0.91$ and $0.61 \mu \mathrm{m})$ and bands $(2)(1.53$ and $0.91 \mu \mathrm{m})$ for the whole trials period, including conditions of poor visibility. The result of this comparison, where a time averaging of the data of 10 minutes and a data sampling period of 5 minutes is applied, is shown in Figure 14. The equation of the overall trend-line: $y=1.043 x-0.132$ shows that the agreement between both sets of retrieved data is quite good. It is noted however, that a second trend-line can be found with a steeper slope below the main trend-line, indicating that the particle density, retrieved with bands (1) is greater than the particle density, retrieved with bands (1). These data points can be allocated to periods of high transmission values (around 0.9 ) for the $1.53 \mu \mathrm{m}$ band. In these cases the slopes for bands (2) are compressed (see for example around 27 March 04.00, Figure 11 above). In a similar way the data above the general trend-line can be allocated to periods with low transmission values for the $0.61 \mu \mathrm{m}$ bands, compressing the slopes in bands (1).

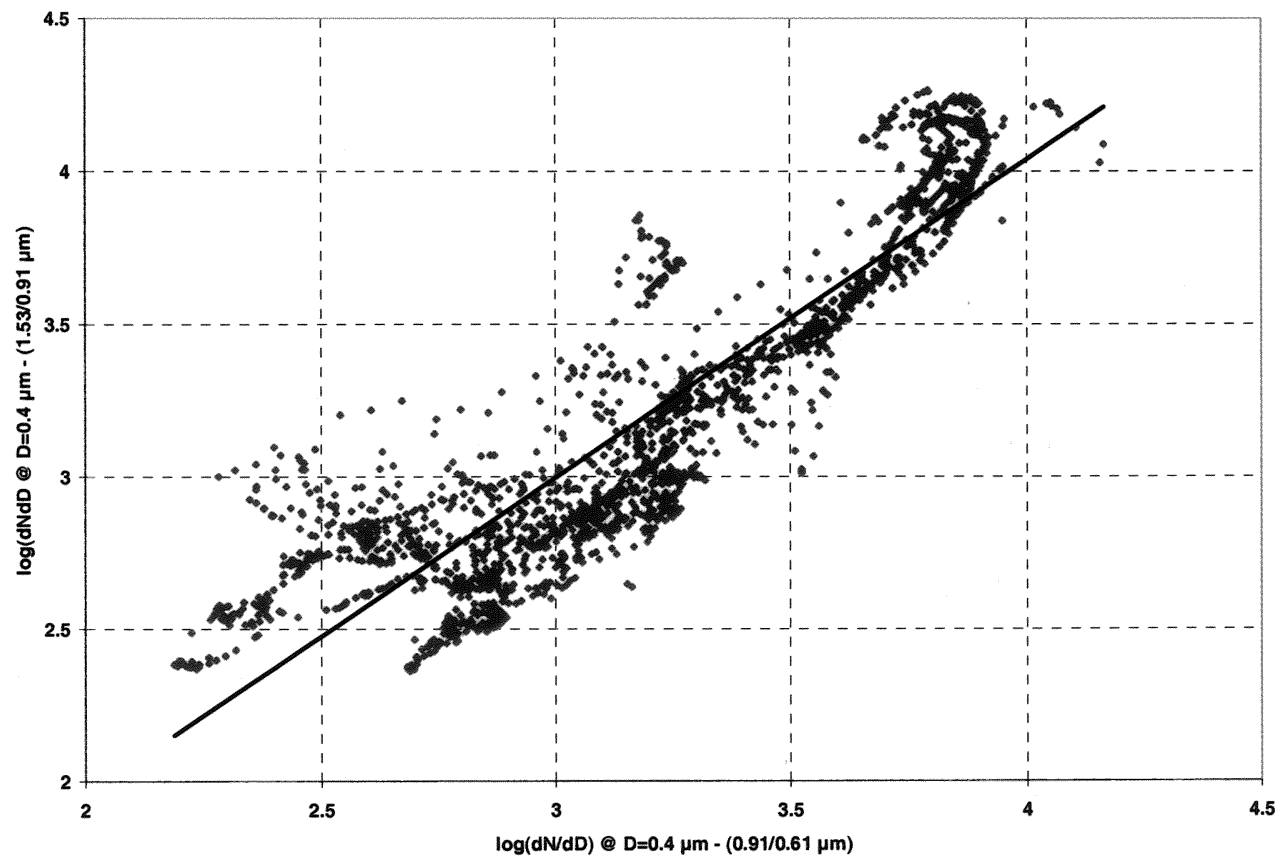

Figure 14. Comparison of particle density $\log (\mathrm{dNdD})$ retrieved from bands (1) (horizontal) and bands (2) (vertical)

\section{DISCUSSION}

In this paper a method to retrieve atmospheric Particle Size Distributions (PSD) from multi-band transmission data has been presented. The method is based upon the assumption, that the particle density is monotonously decreasing with particle diameter. On a logarithmic scale the PSD of atmospheric aerosols approaches in many cases a straight line: the so-called Junge distribution $\log (\mathrm{dN} / \mathrm{dD})=\mathrm{J}_{\mathrm{c}}+\mathrm{J}_{\mathrm{e}}{ }^{*} \log (\mathrm{D})$ where $\mathrm{J}_{\mathrm{c}}$ and $\mathrm{J}_{\mathrm{e}}$ are respectively the Junge coefficient and Junge exponent. For aerosols with PSD's which do not decrease monotonously, the method of retrieving the PSD from spectral transmission data fails because of ambiguities in the relation between the extinction factor and the ratio of particle diameter and wavelength. We assume further, that the number of particles with a diameter greater than $3 \mu \mathrm{m}$ is very low $\left(<1\right.$ per $\mu \mathrm{m}$ per $\left.\mathrm{cm}^{3}\right)$, so that it is attractive to use the visible and near IR spectral bands for the retrieving process. The same is done by investigators of the AERONET network, who use the solar aureole in various spectral bands. 
In the retrieval process, described in this paper, we have taken a number of steps:

- prepare a well calibrated set of multi-band transmission data, preferably between 0.4 and $2 \mu \mathrm{m}$

- eliminate potential bugs in the data due to objects in the measurement path or erroneous background radiance (sun)

- apply time averaging (up to several minutes) in order to reduce low frequency atmospheric refraction effects

- produce for the given path-length relationships between the Junge parameters $\mathrm{J}_{\mathrm{c}}$ and $\mathrm{J}_{\mathrm{e}}$ of hypothetical PSD's and the slope $s(t)$ and average transmission $t(a v)$ for various combinations of two spectral bands: 0.91/0.61 and $1.53 / 0.91 \mu \mathrm{m}$ using formulae (1) and (2), illustrated by Figures 6 and 7

- in this process we assume the aerosols to be spherical, having just the real refractive index of water (about 1.33 ) and having no particles smaller than $0.1 \mu \mathrm{m}$ and bigger than $20 \mu \mathrm{m}$

- apply the measured values of $s(t)$ and $t(a v)$ into these formulae and determine the retrieved $J_{e}$ and $J_{c}$

We have applied the method to the data from the VAMPIRA trials, organized in March/April 2004. In an earlier paper we used the multi-band transmission data from the Multi-Spectral Radiometer Transmissometer (MSRT) system from TNO [8] to analyze the ratio of the extinction coefficient in two spectral bands, leading to conclusions about the nature of the aerosols in the path [1]. For the purpose of this paper we recalibrated the data by selecting a period with extremely good visibility condition and used MODTRAN for the corresponding meteorological conditions in order to separate the extinction by scattering only. During the VAMPIRA trial large variations in visibility did occur, so that a comparison could be made in the applicability of each of the two band combinations (1) and (2). We present values of $J_{e}$ and $J_{c}$, retrieved for several sub-periods for bands (1) and (2) and compare them with $\mathrm{J}_{\mathrm{e}}$ and $\mathrm{J}_{\mathrm{c}}$ values, measured with the TNO Particle Measurement System (PMS) on the Sürendorf shore location. We also compared the particle density for a particle diameter of $0.4 \mu \mathrm{m}$, retrieved via bands (2) and obtained from the PMS system. We conclude that the method of PSD retrieval via multi-band transmission data is very promising. Inherent advantages such as the path-integrated data collection compared to the local particle measurement with the PMS system and the better signal to noise ratio, allowing determination of short term variations in particle density are clearly demonstrated. The minor instrumental problem of large Field of View, causing saturation effects in high background radiance conditions (sun-glints), has been solved in the meantime.

\section{REFERENCES}

[1] Arie N. de Jong et al, Multi-band optical/IR transmissometry during the VAMPIRA trials, spring 2004, SPIE Volume 5572, Optics in Atmospheric Propagation and Adaptive Systems VII, Gran Canaria, September 2004

[2] Arie N. de Jong et al, Investigation of aerosol particle size distribution in the San Diego Bay by means of multi-band transmissometry, SPIE Volume 6303, Atmospheric Optical Modeling, Measurement and Simulation II, San Diego, August 2006

[3] Karin Stein, Investigations on the IR-range performance under different atmospheric conditions, SPIE Volume 5981, Optics in Atmospheric Propagation and Adaptive Systems VIII, Bruges, September 2005

[4] H.C. van de Hulst, Light Scattering by Small Particles, (page 176), Dover Publications, 1981

[5] Arie N. de Jong et al, Long-range transmission at low elevations over the ocean, Proc. Symposium RTO-MP1 on "E-O propagation, signature and system performance under adverse meteorological conditions", Naples, March 1998

[6] F.X. Kneizis et al, The MODTRAN 2/3 report and Lowtran 7 model, Phillips Laboratory, Geophysics Directorate, PL/GPOS, Hanscom AFB, MA 1996

[7] Oleg Dubovik et al, A flexible inversion algorithm for retrieval of aerosol optical properties from sun and sky radiance measurements, Journal of Geophysical Research, Vol. 105, No. D16, 20.673-20.696, August 27, 2000

[8] Arie N. de Jong et al, Description of a 7-channel MSRT system: Multi Spectral Radiometer Transmissometer, TNO report FEL-99-I028, February 1999 